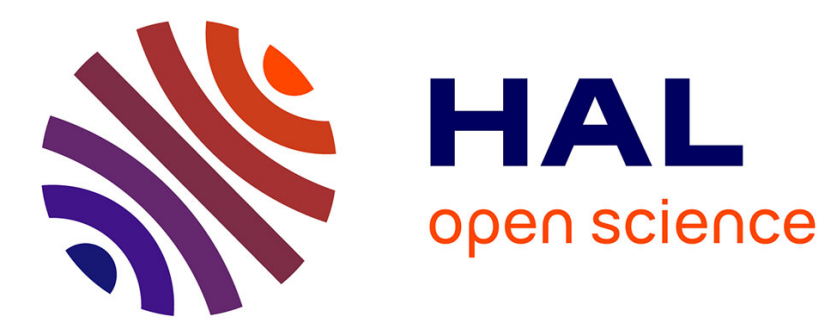

\title{
Performance of a Chirality-affected Channel exhibiting Giant Optical Activity for Terahertz Communications
} Anna Maria Vegni, Valeria Loscrì

\section{To cite this version:}

Anna Maria Vegni, Valeria Loscrì. Performance of a Chirality-affected Channel exhibiting Giant Optical Activity for Terahertz Communications. NanoCom, ACM, Sep 2016, New York, United States. 10.1145/2967446.2967453 . hal-01345407

\section{HAL Id: hal-01345407 https://hal.inria.fr/hal-01345407}

Submitted on 13 Jul 2016

HAL is a multi-disciplinary open access archive for the deposit and dissemination of scientific research documents, whether they are published or not. The documents may come from teaching and research institutions in France or abroad, or from public or private research centers.
L'archive ouverte pluridisciplinaire HAL, est destinée au dépôt et à la diffusion de documents scientifiques de niveau recherche, publiés ou non, émanant des établissements d'enseignement et de recherche français ou étrangers, des laboratoires publics ou privés. 


\section{Performance of a Chirality-affected Channel exhibiting Giant Optical Activity for Terahertz Communications}

\author{
Anna Maria Vegni \\ Department of Engineering \\ COMLAB Telecommunication laboratory \\ Roma Tre University, Rome, Italy \\ annamaria.vegni@uniroma3.it
}

\author{
Valeria Loscrí \\ Inria Lille-Nord Europe \\ FUN Research team \\ Lille, France \\ valeria.Ioscri@inria.fr
}

\begin{abstract}
Terahertz frequency band is an emerging research area related to nano-scale communications. In this frequency range, specific features can provide the possibility to overcome the issues related to the spectrum scarcity and capacity limitation. Apart high molecular absorption, and very high reflection loss that represent main phenomena in $\mathrm{THz}$ band, we can derive the characteristics of the channel affected by chirality effects occurring in the propagation medium, specifically, in the case where a Giant Optical Activity is present. This effect is typical of the so-called chiral-metamaterials in (4-10) $\mathrm{THz}$ band, and is of stimulating interest particularly for millimeter wireless communications.

In this paper, we analyze the behavior of specific parameters of a chiral-metamaterial, like the relative electrical permittivity, magnetic permeability and chirality coefficients, and from that we derive the channel behavior both for Lineof-Sight and No Line-of-Sight propagations. We notice the presence of spectral windows, due to peaks of resonance of chiral parameter. Finally, performances of the chiralityaffected channel have been assessed in terms of $(i)$ channel capacity, (ii) propagation delay, and (iii) coherence bandwidth, for different distances.
\end{abstract}

\section{Keywords}

$\mathrm{THz}$ band, chirality effects, Giant Optical Activity, nanocommunications

\section{INTRODUCTION}

The increasing demand of wireless nanocommunication systems following the framework of Internet of NanoThings [1] has motivated the need to design accurate channel models working in the Terahertz band $[2,3]$. This frequency band represents one of the most promising spectrum bands to enable ultra-high-speed communications, with the aim to overcome the spectrum scarcity and capacity limitations of current wireless systems. Indeed, existing channel mod-

Permission to make digital or hard copies of all or part of this work for personal or classroom use is granted without fee provided that copies are not made or distributed for profit or commercial advantage and that copies bear this notice and the full citation on the first page. Copyrights for components of this work owned by others than ACM must be honored. Abstracting with credit is permitted. To copy otherwise, or republish, to post on servers or to redistribute to lists, requires prior specific permission and/or a fee. Request permissions from permissions@ acm.org.

NANOCOM'16, September 28-30, 2016, New York, NY, USA

(c) 2016 ACM. ISBN 978-1-4503-4061-8/16/09. . $\$ 15.00$

DOI: http://dx.doi.org/10.1145/2967446.2967453 els adopted for lower frequency bands cannot be re-used for $\mathrm{THz}$ communications.

Many researchers have already presented novel channel models for $\mathrm{THz}$ band [4-8], where molecular absorption and spreading loss are the main features studied in this frequency range. Another work [9] considers a multi-ray approach by assuming reflected, scattered and diffracted paths, in order to model the main THz-band channel features, such as the distance-varying spectral windows, and the temporal broadening effects.

As it can be noticed from previous works, the channel modeling in $\mathrm{THz}$ band is addressed through transfer functions that consider specific features, like molecular absorption loss, and spreading loss. However, there are other effects that deserve to be considered and analyzed, such as the electromagnetic chirality effect and the specific features of the propagation medium, that are normally neglected in classical modeling of transmission channels. The relative chirality parameter is a specific characteristic of a chiral homogeneous isotropic medium. Standard values are in the range $[0,1]$, but in particular media such as the chiral complex materials where a Giant Optical Activity (GOA) takes place, the relative chirality parameter is complex and varies with the frequency, showing multiple peaks at specific resonance frequencies [10]. A material that exhibits a GOA and is affected by the chirality effects is called chiralmetamaterial [11]. This is an artificial material that shows a frequency-dependent cross-coupling between the electric and magnetic fields. This interesting property is of great interest to many areas of science, like analytical chemistry and molecular biology.

In this paper, we consider a chiral-metamaterial exhibiting a GOA, and we analyze the specific behavior of channel transfer function in case of direct and multi-path propagation, in the (4-10) $\mathrm{THz}$ frequency range. Due to the resonant behavior of chiral parameter, the channel model shows specific frequency-dependent spectral windows, guaranteeing high bandwidth values.

This paper is organized as follows. In Section 2 we introduce the frequency-dependent behavior of specific parameters of a chiral-metamaterial. Starting from the concept of electromagnetic chirality [12-14], we consider the chiral effects following the change in the propagation velocity and in the refractive index, due to the chiral impurities inside the propagation medium. These effects are evaluated also in the case when the considered medium exhibits a GOA [10,15]. Then, in Section 3 we derive the corresponding chiralityaffected channel model for $\mathrm{THz}$ band, and present the re- 


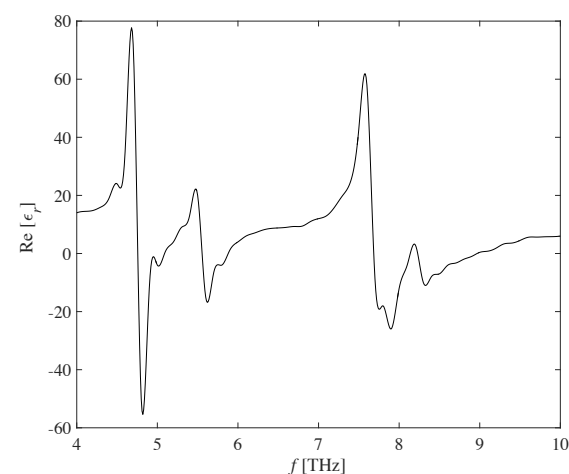

(a)

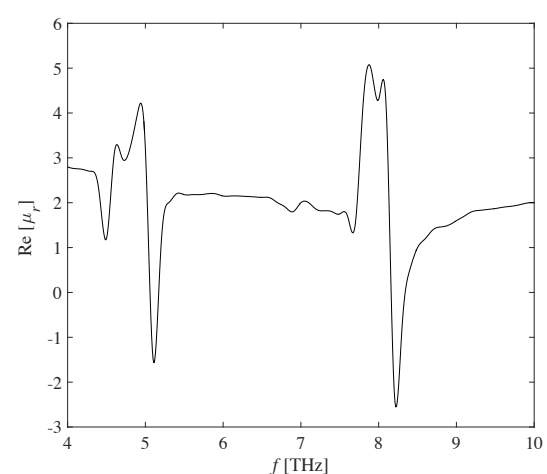

(b)

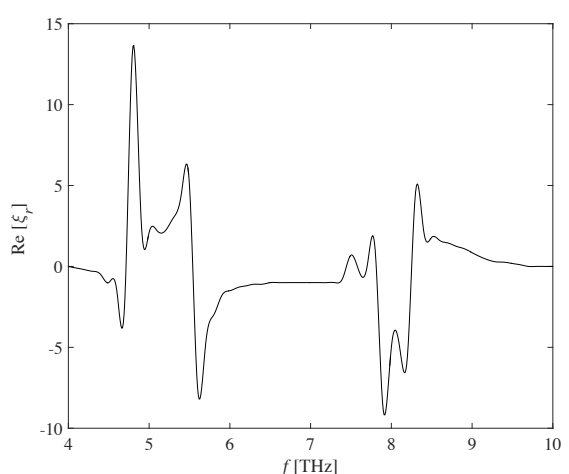

(c)

Figure 1: Resonant frequency-dependent behavior of relative $(a)$ electrical permittivity, $(b)$ magnetic permeability, and (c) chirality parameter, in the THz band from 4 to $10 \mathrm{THz},[10]$.

lated frequency-dependent spectral windows. Section 4 is then devoted to the performances of the chiral channel, assessed in terms of capacity, propagation delay, and coherence bandwidth, in case of LoS and NLoS propagation modes. Finally, conclusions are drawn at the end of the paper.

\section{FULL-WAVE PROPAGATION IN A CHIRAL-METAMATERIAL}

This section is devoted to the design of a full-wave propagation model for $\mathrm{THz}$ band, when a GOA is working. Starting from the classic harmonic macroscopic Maxwell's equations, we consider the electromagnetic propagation inside a generic complex material, under the assumption that it is a linear and chiral medium.

Unconventional materials (i.e. metamaterials) are specifically considered since the GOA is reinforced by using thin metallic crossed-structure impurities in the host dielectric medium, that is chiral-metamaterials [11]. Therefore, we will consider a time-harmonic generic linear material, where chiral (magneto/electric-optical) effects are included in the following constitutive relations, that can be written as:

$$
\left\{\begin{array}{l}
\mathbf{B}=\underline{\xi} \bullet \mathbf{E}+\underline{\mu} \bullet \mathbf{H} \\
\mathbf{D}=\underline{\varepsilon} \bullet \mathbf{E}+\underline{\zeta} \bullet \mathbf{H}
\end{array}\right.
$$

where we remind that $\mathbf{B}$ is the magnetic displacement and $\mathbf{H}$ is the magnetic field, as well as $\mathbf{D}$ is the electric displacement and $\mathbf{E}$ is the electric field. Finally, the symbol $\bullet$ represents the scalar product operator, and $\underline{\xi}, \mu, \underline{\varepsilon}$, and $\zeta$ are specific tensor quantities of the material.

From (1) we observe the chirality property through the dependence of $(i) \mathbf{E}$ in $\mathbf{B}$, and $(i i) \mathbf{H}$ in $\mathbf{D}$. Furthermore, the displacement field existing inside the material is generated by an excitation expressed in terms of intensity of the incident electro-magnetic field. Therefore, the material under consideration is a linear chiral medium.

We remind that the chiral effects have a two-fold meaning i.e., $(i)$ an electric field applied on the material provides not only an electric induction, but also a magnetic displacement, and (ii) a magnetic field applied on the material provides not only a magnetic induction, but also an electric displacement, unlikely from other non-chiral materials.

As previously said, in this paper our attention is devoted to a particular class of chiral-metamaterials (GOA). In [16], the authors show that for a GOA reciprocal material the specific constitutive relations are as follows:

$$
\left[\begin{array}{l}
\mathbf{B} \\
\mathbf{D}
\end{array}\right]=\left[\begin{array}{cc}
-j \frac{\xi_{0} \xi_{r}}{c} & \mu_{0} \mu_{r} \\
\varepsilon_{0} \varepsilon_{r} & j \frac{\xi_{0} \xi_{r}}{c}
\end{array}\right] \bullet\left[\begin{array}{c}
\mathbf{E} \\
\mathbf{H}
\end{array}\right]
$$

where it is clear that the natural dielectric (where $\varepsilon_{b}$ and $\mu_{b}$ are the permittivity and permeability, respectively) becomes a metamaterial. Moreover, for a GOA material, at the frequency around $5 \mathrm{THz}$ and $8 \mathrm{THz}$, there are four resonance frequencies of the $\xi_{r}$ relative chirality parameter i.e., $[4.8,5.6,7.9,8.2] \mathrm{THz}$.

The same consideration is applied to the relative permittivity and permeability parameters, still in the $(4-10) \mathrm{THz}$ band. As reported in [10], $\varepsilon_{r}$ and $\mu_{r}$ are complex parameters, and the real part has a frequency-dependent behavior, with resonant peaks. Figure $1(a),(b)$, and $(c)$ depict the trends of real parts of $\varepsilon_{r}, \mu_{r}$, and $\xi_{r}$, respectively, where it is easy to observe the resonant peaks.

Equation (2) becomes

$$
\begin{aligned}
& {\left[\begin{array}{l}
\mathbf{B} \\
\mathbf{D}
\end{array}\right]=} \\
& =\left[\begin{array}{cc}
-j \frac{\Omega_{\xi} \omega_{0} \omega}{\omega_{0}^{2}-\omega^{2}-j \omega \gamma} & \mu_{0}\left(\mu_{b}+\frac{\Omega_{\mu} \omega^{2}}{\omega_{0}^{2}-\omega^{2}-j \omega \gamma}\right) \\
\varepsilon_{0}\left(\varepsilon_{b}+\frac{\Omega_{\varepsilon} \omega_{0}^{2}}{\omega_{0}^{2}-\omega^{2}-j \omega \gamma}\right) & j \frac{\Omega_{\xi} \omega_{0} \omega}{\omega_{0}^{2}-\omega^{2}-j \omega \gamma}
\end{array}\right] \bullet\left[\begin{array}{c}
\mathbf{E} \\
\mathbf{H}
\end{array}\right],
\end{aligned}
$$

where $\omega=2 \pi f, \varepsilon_{0}$, and $\mu_{0}$ are the absolute permittivity and permeability, respectively. Then, the constitutive parameters of interest are:

$$
\begin{gathered}
\varepsilon=\varepsilon_{0} \varepsilon_{r}=\varepsilon_{0}\left[\varepsilon_{b}+\Omega_{\varepsilon} \omega_{0}^{2}\left(\frac{\omega_{0}^{2}-\omega^{2}+j \omega \gamma}{\left(\omega_{0}^{2}-\omega^{2}\right)^{2}+\omega^{2} \gamma^{2}}\right)\right], \\
\mu=\mu_{0} \mu_{r}=\mu_{0}\left[\mu_{b}+\Omega_{\mu} \omega^{2}\left(\frac{\omega_{0}^{2}-\omega^{2}+j \omega \gamma}{\left(\omega_{0}^{2}-\omega^{2}\right)^{2}+\omega^{2} \gamma^{2}}\right)\right], \\
\xi_{r}=\Omega_{\xi} \omega_{0} \omega\left(\frac{\omega_{0}^{2}-\omega^{2}+j \omega \gamma}{\left(\omega_{0}^{2}-\omega^{2}\right)^{2}+\omega^{2} \gamma^{2}}\right) .
\end{gathered}
$$

It is noted that the frequency resonant behavior of $\xi_{r}$ is a like-Drude one, and $\Omega_{\varepsilon}, \Omega_{\mu}, \Omega_{\xi}, \gamma$, and $\omega_{0}$ are specific parameters of the GOA material [16].

By simple computations, and assuming a lossy material 
i.e.,

$$
\left\{\begin{array}{l}
\varepsilon_{b}=\operatorname{Re}\left[\varepsilon_{b}\right]+j \operatorname{Im}\left[\varepsilon_{b}\right] \\
\mu_{b}=\operatorname{Re}\left[\mu_{b}\right]+j \operatorname{Im}\left[\mu_{b}\right]
\end{array}\right.
$$

we obtain

$$
\begin{gathered}
\varepsilon_{r}=\left(\operatorname{Re}\left[\varepsilon_{b}\right]+\frac{\omega_{0}^{2}\left(\omega_{0}^{2}-\omega^{2}\right) \Omega_{\varepsilon}}{\left(\omega_{0}^{2}-\omega^{2}\right)^{2}+\omega^{2} \gamma^{2}}\right)+ \\
j\left(\frac{\gamma \omega_{0}^{2} \Omega_{\varepsilon} \omega}{\left(\omega_{0}^{2}-\omega^{2}\right)^{2}+\omega^{2} \gamma^{2}}+\operatorname{Im}\left[\varepsilon_{b}\right]\right) \\
\mu_{r}=\left(\operatorname{Re}\left[\mu_{b}\right]+\frac{\omega^{2}\left(\omega_{0}^{2}-\omega^{2}\right) \Omega_{\mu}}{\left(\omega_{0}^{2}-\omega^{2}\right)^{2}+\omega^{2} \gamma^{2}}\right)+ \\
j\left(\frac{\gamma \Omega_{\mu} \omega^{3}}{\left(\omega_{0}^{2}-\omega^{2}\right)^{2}+\omega^{2} \gamma^{2}}+\operatorname{Im}\left[\mu_{b}\right]\right), \\
\xi_{r}=\omega_{0} \Omega_{\xi}\left[\frac{\omega\left(\omega_{0}^{2}-\omega^{2}\right)}{\left(\omega_{0}^{2}-\omega^{2}\right)^{2}+\omega^{2} \gamma^{2}}\right]+j \omega_{0} \Omega_{\xi}\left[\frac{\gamma \omega^{2}}{\left(\omega_{0}^{2}-\omega^{2}\right)^{2}+\omega^{2} \gamma^{2}}\right] .
\end{gathered}
$$

Now, by posing the following expression for the square relative refractive index:

$$
\begin{aligned}
& \varepsilon_{r} \mu_{r}+\xi_{r}^{2}=\left(\operatorname{Re}\left[\varepsilon_{r}\right]+j \operatorname{Im}\left[\varepsilon_{r}\right]\right)\left(\operatorname{Re}\left[\mu_{r}\right]+j \operatorname{Im}\left[\mu_{r}\right]\right)+ \\
& +\left(\operatorname{Re}\left[\xi_{r}\right]+j \operatorname{Im}\left[\xi_{r}\right]\right)^{2}>0,
\end{aligned}
$$

we observe that the following conditions hold for a GOA material:

$$
\begin{gathered}
\Omega_{\varepsilon}=\left(1+\frac{\gamma^{2}}{\omega^{2}-\omega_{0}^{2}}\right) \operatorname{Re}\left[\varepsilon_{b}\right], \\
\Omega_{\mu}=\left(1+\frac{\gamma^{2}}{\omega^{2}-\omega_{0}^{2}}\right) \operatorname{Re}\left[\mu_{b}\right], \\
\Omega_{\xi}=\left(1+\frac{\gamma^{2}}{\omega^{2}-\omega_{0}^{2}}\right) \sqrt{\operatorname{Re}\left[\varepsilon_{b}\right] \operatorname{Re}\left[\mu_{b}\right]} .
\end{gathered}
$$

It is observed that relations (12), (13), and (14) state the connection between the host material parameters i.e., $\varepsilon_{b}$, and $\mu_{b}$, and the specific GOA ones in order to obtain a positive refractive index in (11).

Finally, the computation of the electro-magnetic field in the channel is carried out through the solutions of linear differential equations, arising from the following source-less Maxwell's equations:

$$
\left\{\begin{array}{l}
(\underline{\nabla}+j \omega \underline{\xi}) \bullet \mathbf{E}=-j \omega \mu \bullet \mathbf{H} \\
(\underline{\nabla}-j \omega \underline{\zeta}) \bullet \mathbf{H}=j \omega \underline{\varepsilon} \bullet \mathbf{E}
\end{array}\right.
$$

and the solving differential equations for $\mathbf{E}$ and $\mathbf{H}$ are given, respectively:

$$
\left\{\begin{array}{l}
{\left[(\underline{\nabla}-j \omega \underline{\zeta}) \bullet \underline{\mu}^{-1} \bullet(\underline{\nabla}+j \omega \underline{\xi})-\omega^{2} \underline{\varepsilon}\right] \bullet \mathbf{E}=0} \\
{\left[(\underline{\nabla}+j \omega \underline{\xi}) \bullet \underline{\varepsilon}^{-1} \bullet(\underline{\nabla}-j \omega \underline{\zeta})-\omega^{2} \underline{\mu}\right] \bullet \mathbf{H}=0}
\end{array}\right.
$$

where $\nabla$ is Kong's operator.

Finally, by assuming the linear polarization of the electric field (i.e., $\mathbf{E}=E_{x} \hat{\mathbf{x}}$ ), we obtain the final differential equation for $E_{x}$, i.e.

$\alpha_{1} E_{x}+\alpha_{2} \frac{\partial E_{x}}{\partial x}+\alpha_{3} \frac{\partial E_{x}}{\partial y}+\alpha_{4} \frac{\partial E_{x}}{\partial z}+\alpha_{5} \frac{\partial^{2} E_{x}}{\partial y^{2}}+\alpha_{6} \frac{\partial^{2} E_{x}}{\partial z^{2}}=0$,

where $\alpha_{i}$ with $i=(1,2, \ldots, 6)$ are coefficients depending on the elements of $\underline{\varepsilon}, \underline{\mu}, \underline{\xi}$, and $\underline{\zeta}$ tensors.

\section{CHIRAL CHANNEL MODEL}

In this section, we present how the relative chiralilty parameter affects the channel performance in the $(4-10) \mathrm{THz}$ band, in the case of ray tracing propagation (i.e., LoS, and NLoS), and under the linear polarization hypothesis. Specifically, in NLoS case, we focus on reflected paths due at generic reflection centers located at $z$-plane. The reflection characteristics of the transmissive channel can be evaluated through the specific knowledge of the local planar geometry associated to the reflection centers.

The use of ray tracing techniques for channel modeling in $\mathrm{THz}$ band has been largely adopted, like in [7], where Han et al. consider a multi-ray approach with one direct path, and other reflected, scattered, and diffracted paths. According to this approach, the channel model is the combination of several individual narrow sub-bands, each of them with a flat-band response. Assuming $N_{i}$ narrow sub-bands, and in the case of stationary environment, the channel response in the $i$-th sub-band is given as

$$
h_{i}(\tau)=\sum_{n=1}^{N_{i}} \alpha_{i, n} \delta\left(\tau-\tau_{n}\right)
$$

where $\alpha_{i, n}$ is the frequency-dependent attenuation, and $\tau_{n}$ is the propagation delay of the $n$-th ray in the multi-ray approach.

From (18), and according to the computations in [7], we can derive the LoS and NLoS channel transfer functions in the case of chirality-affected channel with GOA, respectively as:

$$
H_{\mathrm{LoS}}(f)=H_{\mathrm{Abs}}(f) H_{\mathrm{Spr}}(f) e^{-j 2 \pi f \tau_{\mathrm{LoS}}},
$$

and

$H_{\mathrm{NLoS}}(f)=\left[\frac{\nu_{c}}{4 \pi f\left(d_{1}+d_{2}\right)}\right] e^{-j 2 \pi f \tau_{\mathrm{NLoS}}-\frac{1}{2} k(f)\left(d_{1}+d_{2}\right)} \cdot R(f)$,

where we assume the NLoS scenario is affected by reflected rays only, through the rough surface reflection loss i.e., $R(f)$.

In (19), $H_{\mathrm{Abs}}$ is the transfer function due to the molecular absorption loss, while $H_{\mathrm{Spr}}$ is the spreading loss that takes account for the chirality effect through $\nu_{c}$ that is the propagation velocity of the electro-magnetic field in a chiral homogeneous isotropic medium i.e.,

$$
\nu_{c}=\frac{c}{n_{c}}
$$

where $c$ is the light propagation speed, and $n_{c}$ is the refractive index in a chiral medium i.e.,

$$
n_{c}=\sqrt{\mu_{r} \varepsilon_{r}+\xi_{r}^{2}},
$$

with $\mu_{r}, \varepsilon_{r}$ and $\xi_{r}$ frequency-dependent parameters, according to Figure 1. Finally, under the hypothesis of stationary scenario where the transmitter and the receiver are at a distance $d[\mathrm{~m}]$, from (19) we obtain the propagation delay for the LoS ray as:

$$
\tau_{\mathrm{LoS}}=\frac{d}{\nu_{c}} .
$$

For the NLoS channel transfer function expressed in (20), by assuming $d_{1}$ as the distance between the transmitter and a generic reflecting point, and $d_{2}$ as the distance between this point and the receiver, we obtain the propagation delay 


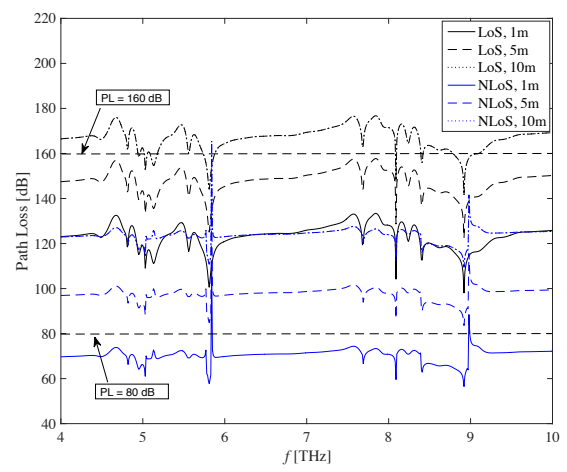

(a)

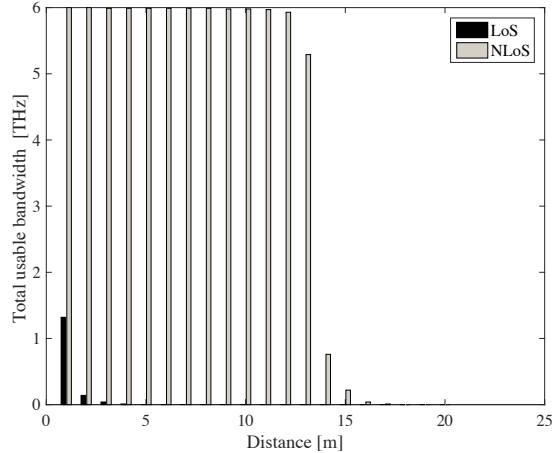

(b)

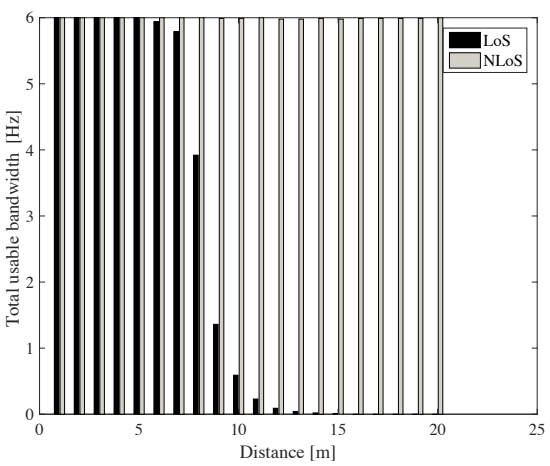

$(c)$

Figure 2: Spectral windows in a chiral-affected channel with GOA. (a) Path loss, and (b) total usage bandwidth for LoS and NLoS propagation in case of a path loss threshold of $120 \mathrm{~dB}$, and (c) $160 \mathrm{~dB}$.

of the $j$-th NLoS ray along the distance $\left(d_{1}+d_{2}\right)$, as

$$
\tau_{\mathrm{NLOS}}=\frac{d_{1}+d_{2}}{\nu_{c}} .
$$

From the expressions of channel transfer functions in (19) and (20), it is easy to compute the total path loss, as depicted in Figure $2(a)$ in case of LoS and NLoS propagations, for different distances from transmitter to receiver, and assuming a specific reflecting angle for multi-path. By increasing the distance, the path loss has a higher trend. Moreover, similarly to the results in [9], the LoS propagation (black lines) provides higher values with respect to the NLoS scenario (blue lines). In both LoS and NLoS, the behavior is frequency-dependent, but in LoS propagation the path loss has a smoother trend, with some peaks at 5.8, 8.09 and $8.93 \mathrm{THz}$. On the other hand, for NLoS propagation, the peaks are well noticeable at 5.8 and $8.93 \mathrm{THz}$, while on the other frequencies, the trend is on average flat around 70 , 100 and $120 \mathrm{~dB}$ for $\operatorname{LoS}$ at $d=1,5$, and $10 \mathrm{~m}$, respectively.

Similarly to the analysis conducted in [9], we aim to characterize the spectral windows of chiral channel transfer functions in case of LoS and NLoS propagation. A spectral window is given by the portion of spectrum below a given path loss threshold. We expect to observe that the path loss peaks caused by the chiral effect create several spectral windows, with different bandwidths in each of them.

In the case of a path loss threshold set to $80 \mathrm{~dB}$, the communication distance is limited for NLoS propagation at lower distance of $1 \mathrm{~m}$, except the two peaks that are above this threshold, and correspond to $5.84 \mathrm{THz}$ and $8.98 \mathrm{THz}$. According to the values assumed in [9], the threshold of $80 \mathrm{~dB}$ corresponds to no gains of transmission and reception antennas, and so to a multi-path propagation model.

In order to identify the spectral windows for LoS propagation, we have to increase the path loss threshold around $120 \mathrm{~dB}$, so that a few windows appear for a distance of $1 \mathrm{~m}$ (see curve LoS for $d=1 \mathrm{~m}$ in Figure $2(a)$ ). However, in this case most of the path loss for LoS propagation is above the threshold, thus providing a reduced usable bandwidth. Figure $2(b)$ depicts the usable bandwidth versus the distance for the path loss threshold of $120 \mathrm{~dB}$. We notice that the available spectrum in LoS propagation is limited up to $4 \mathrm{~m}$, reaching a maximum at $1.32 \mathrm{THz}$ corresponding to a distance of $1 \mathrm{~m}$. In contrast, the NLoS propagation reaches higher bandwidths, and then it decreases at $17 \mathrm{~m}$ where the bandwidth is $0.01 \mathrm{THz}$. The bandwidth rate in LoS is $75.5 \mathrm{GHz} / \mathrm{m}$, while it reaches $3.90 \mathrm{THz} / \mathrm{m}$ in NLoS scenario.

It follows that an increase of the path loss threshold to $160 \mathrm{~dB}$ is expected to provide higher values of usable bandwidth in LoS propagation, as depicted in Figure $2(c)$. Higher path loss thresholds rise from higher antenna gains, and the transmission becomes directional through the LoS path. In this case, the usage bandwidth in LoS propagation reaches higher values than those for the threshold of $120 \mathrm{~dB}$. The lowest value is $0.01 \mathrm{THz}$ for a distance of $15 \mathrm{~m}$, and the average bandwidth rate for LoS propagation is $2.39 \mathrm{THz} / \mathrm{m}$.

On the other side, for NLoS propagation, the usable bandwidth reaches approximately the maximum value of $6 \mathrm{THz}$ for different distances, and then the average rate of the total usable bandwidth is $5.99 \mathrm{THz} / \mathrm{m}$. As a result, we can conclude that within the range $(4-10) \mathrm{THz}$ the available bandwidth is almost the entire band, especially for NLoS propagation.

\section{CHIRAL CHANNEL CHARACTERIZA- TION}

Following the chirality-affected channel model presented in Section 3, in this section we investigate its main features in the (4-10) $\mathrm{THz}$ band. Specifically, we aim to characterize (i) the channel capacity, (ii) the propagation delay, and ( $i i i)$ the coherence bandwidth.

\subsection{Channel capacity and propagation delay}

To evaluate the capacity limits in a chiral medium, we refer to the approach adopted in [7], where the received signal has been decomposed as a sum of the sub-bands, each one with a narrow behavior and a flat-band response. The following constraint is adopted:

$$
\sum_{i=1}^{N_{B}} P_{i} \leq P_{\text {TOT }},
$$

where $N_{B}$ is the total number of sub-bands, $P_{i}$ is the transmission power in the $i$-th sub-band, and $P_{T O T}$ is the total transmit power in the $(4-10) \mathrm{THz}$ band. Notice that, since the chiral parameter has a frequency-dependent behavior in (4-10) THz band, we consider only this frequency range. 


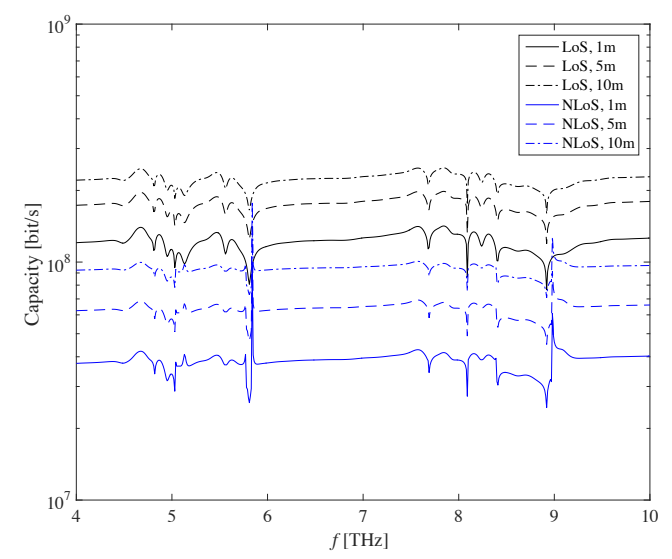

Figure 3: Capacity of a chirality-affected channel with GOA, versus frequency for LoS and NLoS propagation, and different distances.

For $N_{B}$ sub-bands, the capacity can be defined as the sum of the single capacities in each sub-band i.e.,

$$
C=\sum_{i=1}^{N_{B}} C_{i}=\sum_{i=1}^{N_{B}} \Delta f_{i} \log \left(1+\frac{\left|h_{i}\right|^{2} P_{i}}{\Delta f_{i} S_{N}\left(f_{i}\right)}\right),
$$

where $S_{N}$ is the power spectral density of the additive white Gaussian noise, and $\Delta f_{i}$ is the sub-band range among two consecutive sub-bands i.e., $\Delta f_{i}=f_{i+1}-f_{i}$, assumed as $10 \mathrm{GHz}$ in our simulation results. We assume a flat power profile, that is the total power transmission is uniformly distributed over the entire operative band (i.e., from 4 to $10 \mathrm{THz}$ ). Also, we consider a power level of $46 \mathrm{dBm}$ in all the sub-bands i.e., $N_{B}=600$.

Figure 3 depicts the chirality-affected channel capacity with GOA in case of LoS and NLoS scenario. We notice that with a reduction of distance, the capacity decreases as well, and also the LoS scenario has a smoother behavior with respect to the frequency, while the NLoS shows an accentuate frequency-dependent trend, with distinguishable peaks at resonance frequencies. Specifically, in LoS, the capacity has an almost flat behavior, with a mean value of $0.22 \mathrm{Gbit} / \mathrm{s}$ for $d=10 \mathrm{~m}$. Performances get worst in the case of NLoS propagation for $d=1 \mathrm{~m}$, where we observe a degradation of capacity at 5.81 and $8.92 \mathrm{THz}$, corresponding to $25.65 \mathrm{Mbit} / \mathrm{s}$ and $24.47 \mathrm{Mbit} / \mathrm{s}$, respectively.

From the expressions in (23) and (24), the propagation delay in LoS and NLoS scenarios is depicted in Figure 4. We observe the frequency-dependent behavior due to the chiral effect, and as expected, performance gets worst when the distance increases. An almost-flat behavior is shown for LoS at short distances (i.e., $d=1 \mathrm{~m}$ ), while a resonant trend appears when increasing the distance, as well as in NLoS scenario due to the longer distances covered. Finally, we observe that the propagation delay both in LoS and NLoS case shows lower values corresponding to 5.81 and $8.92 \mathrm{THz}$.

\subsection{Coherence bandwidth}

The root mean square (rms) delay spread is a measure of how dispersive the channel is. It is expressed as [9]:

$$
\sigma_{i}=\sqrt{\overline{\tau_{i}^{2}}-\bar{\tau}_{i}^{2}}
$$

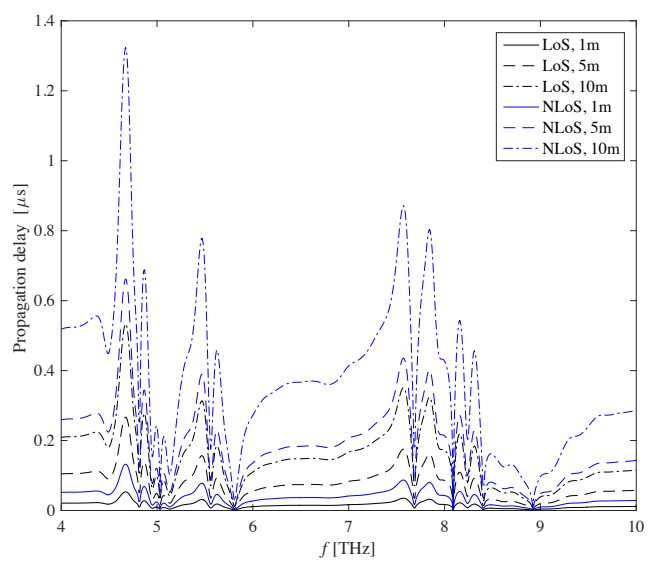

Figure 4: Propagation delay versus frequency in a chirality-affected channel with GOA, for LoS and NLoS propagation, and different distances.

where $\overline{\tau_{i}}$ and $\bar{\tau}_{i}^{2}$ are the first and second moments of the instantaneous power-delay profile, respectively. From (27) we can derive information about the coherence bandwidth, defined as the range of frequencies over which the channel correlation exceeds $50 \%$.

In our simulations, we consider two scenarios with a variable number of NLoS reflected rays i.e., ( $i$ ) one, and (ii) five, and one direct ray. In both cases, we observe the frequencydependent behavior as typical of chiral materials exhibiting GOA. In Figure $5(a)$ we show the coherence bandwidth in the case of one LoS and one NLoS path for different distances. As experienced in [7], higher values are reached for shorter distances. However, we cannot compare our results to others obtained with pre-existing approaches, since the frequency range is not the same.

In our simulations, several peaks appear due to the chirality effects. This can allow tuning the frequency to resonant peaks in order to obtain higher performances. For example, for $d=1 \mathrm{~m}$, the minimum value of rms delay is $0.33 \mathrm{~ns}$, corresponding to $8.92 \mathrm{THz}$. This value corresponds to a symbol rate limited to $0.1 / \sigma_{i}=0.29 \mathrm{Gbit} / \mathrm{s}$ to avoid inter-symbol interference. Also, in this case, the coherence bandwidth is limited to $0.59 \mathrm{GHz}$ at the frequency peak of $8.92 \mathrm{THz}$. On the other side, when the distance increases (i.e., $d=10 \mathrm{~m}$ ), the minimum rms delay is $3.6 \mathrm{~ns}$ at $8.92 \mathrm{THz}$. This value provides a symbol rate limited to $27.71 \mathrm{Mbit} / \mathrm{s}$, and the coherence bandwidth equals to $55.43 \mathrm{MHz}$, still at the same frequency.

Performances get worst in case of multiple reflected paths, as shown in Figure $5(b)$. For $d=1 \mathrm{~m}$ the minumum rms is $0.78 \mathrm{~ns}$, which corresponds to a symbol rate of $0.12 \mathrm{Gbit} / \mathrm{s}$ and a coherence bandwidth of $0.25 \mathrm{GHz}$. For higher distances (i.e., $d=10 \mathrm{~m}$ ) the minimum $\mathrm{rms}$ is $7.31 \mathrm{~ns}$, corresponding to a coherence bandwidth is $27.3 \mathrm{MHz}$ and symbol rate of $13.6 \mathrm{Mbit} / \mathrm{s}$.

\section{CONCLUSIONS}

In this paper, we have presented the performance of a chirality-affected channel with GOA, under the hypothesis of LoS and NLoS propagation in the $(4-10) \mathrm{THz}$ band. We considered the effects of the relative chiral parameter, assuming a frequency-dependent behavior with resonant peaks 


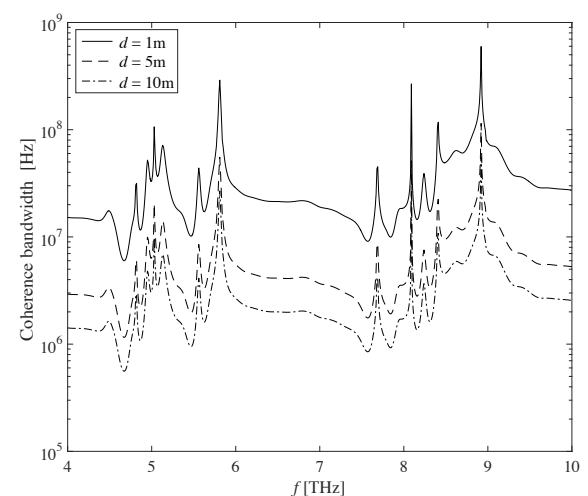

(a)

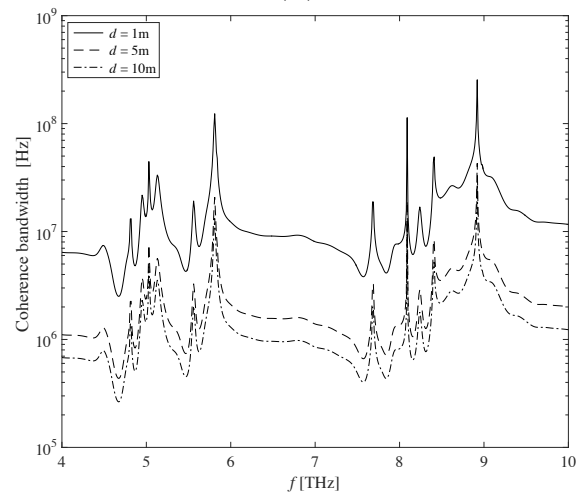

(b)

Figure 5: Coherence bandwidth in a chiralityaffected channel with GOA, in case of $(a)$ one LoS and one NLoS path, an $(b)$ one LoS and five NLoS paths.

at specific frequencies. As a result, this affects the channel transfer function, as well as other performances. In particular, we identified the spectral windows that rise from the chiral effect, and the associated usable bandwidths. The spectral windows vary with the distance and the frequency, with corresponding bandwidths up to $6 \mathrm{THz}$, both in LoS and NLoS propagation. Other results allows to identify specific frequencies that guarantee high performances. As an instance, the rms delay is dependent on the distance and carrier frequency, and reaches minimum values at $8.92 \mathrm{THz}$, corresponding to higher coherence bandwidths.

We can conclude that this particular material exhibiting GOA can guarantee high performances, especially at lower distances. Also, distance-adaptive and multi-carrier transmissions represent the more appropriate communication techniques that can benefit from the relationship between distance and bandwidth in the range (4-10) THz.

\section{REFERENCES}

[1] I. F. Akyildiz and J. M. Jornet. The Internet of Nano-Things. IEEE Wireless Communication Magazine, 17(6):58-63, December 2010.

[2] R. Piesiewicz, T. Kleine-Ostmann, N. Krumbholz, D. Mittleman, J. Koch, and T. Kurner. Short-range ultra-broadband terahertz communications: Concepts and perspectives. Antennas and Propagation
Magazine, IEEE, 49(6):24-39, Dec 2007.

[3] I. F. Akyildiz, J. M. Jornet, and C. Han. Terahertz Band: Next Frontier for Wireless Communications. Physical Communication (Elsevier) Journal, 12:16-32, 2014.

[4] J.M. Jornet and I.F. Akyildiz. Channel modeling and capacity analysis for electromagnetic wireless nanonetworks in the terahertz band. Wireless Communications, IEEE Transactions on, 10(10):3211-3221, Oct. 2011.

[5] I. Llatser, A. Mestres, S. Abadal, E. Alarcon, Heekwan Lee, and A. Cabellos-Aparicio. Time- and frequency-domain analysis of molecular absorption in short-range terahertz communications. Antennas and Wireless Propagation Letters, IEEE, 14:350-353, 2015.

[6] G. Piro, Ke Yang, G. Boggia, N. Chopra, L.A. Grieco, and A. Alomainy. Terahertz communications in human tissues at the nanoscale for healthcare applications. Nanotechnology, IEEE Transactions on, 14(3):404-406, May 2015.

[7] C. Han, A.O. Bicen, and I.F. Akyildiz. Multi-ray channel modeling and wideband characterization for wireless communications in the terahertz band. Wireless Communications, IEEE Transactions on, 14(5):2402-2412, May 2015.

[8] C. Zhang, C. Han, and I. F. Akyildiz. Three dimensional end-to-end modeling and directivity analysis for graphene-based antennas in the terahertz band. In 2015 IEEE Global Communications Conference (GLOBECOM), pages 1-6, Dec 2015.

[9] C. Han, A.O. Bicen, and I.F. Akyildiz.

Multi-wideband waveform design for distance adaptive wireless communications in the terahertz band. Signal Processing, IEEE Transactions on, 64(4):910-922, Febr. 2016.

[10] F. Fang and Y. Cheng. Dual-band terahertz chiral metamaterial with giant optical activity and negative refractive index based on cross-wire structure. Progress In Electromagnetics Research M, 31:59-69, 2013.

[11] B. Wang, T. Koschny, M. Kafesaki, and C. M. Soukoulis. Chiral metamaterials: Simulations and experiments. J. Opt. A: Pure Appl. Opt., 11, 2009.

[12] N. Engheta. Chiral Materials and Chiral Electrodynamics: Background \& Basic Physical Principles. In Special Workshop on Chiral and Complex Materials Progress in Electromagnetics Research Symposium, July 1991.

[13] A. Lakhtakia. Recent contributions to classical electromagnetic theory of chiral media: what next? Speculations in Science and Technology, 14(1):2-17, 1991.

[14] I.V. Lindell, A.H. Sihvola, S.A. Tretyakov, and A.J. Viitanen. Electromagnetic waves in chiral and bi-isotropic media. Artech House, 1994.

[15] J. Zhou, D. R. Chowdhury, R. Zhao, A.K. Azad, H.-T. Chen, C. M. Soukoulis, and A. J. Taylor. Terahertz chiral metamaterials with giant and dynamically tunable optical activity. Phys. Rev. B, 86, Jul 2012.

[16] R. Zhao, T. Koschny, and C. M. Soukoulis. Chiral metamaterials: retrieval of the effective parameters with and without substrate. Optics express, 18(4), July 2010. 